\title{
PROJECTIVELY FULL IDEALS IN NOETHERIAN RINGS, A SURVEY
}

\author{
Catalin Ciuperca, William Heinzer, Jack Ratliff and David Rush
}

November 18, 2018

\begin{abstract}
We discuss projective equivalence of ideals in Noetherian rings and the existence or failure of existence of projectively full ideals. We describe connections with the Rees valuations and Rees integers of an ideal, and consider the question of whether improvements can be made by passing to an integral extension ring.
\end{abstract}

\section{Definitions, summary and examples.}

Let $R$ be a Noetherian ring and let $I \neq R$ be a regular ideal of $R$. (So $I$ contains an element with zero annihilator.)

Definition 1.1 An ideal $J$ of $R$ is projectively equivalent to $I$ if there exist positive integers $m$ and $n$ such that $I^{m}$ and $J^{n}$ have the same integral closure, i.e., $\left(I^{m}\right)_{a}=\left(J^{n}\right)_{a}$.

Projective equivalence is an equivalence relation on the regular proper ideals of $R$. Let $\mathbf{P}(I)$ denote the set of integrally closed ideals projectively equivalent to $I$.

Remark 1.2 The set $\mathbf{P}(I)$ is discrete and linearly ordered with respect to inclusion. Moreover, $J$ and $K$ in $\mathbf{P}(I)$ and $m$ and $n$ positive integers implies $\left(J^{m} K^{n}\right)_{a} \in \mathbf{P}(I)$. Thus there is naturally associated to $I$ and $\mathbf{P}(I)$ a unique subsemigroup $S(I)$ of the additive semigroup of nonnegative integers $\mathbb{N}_{0}$ such that $S(I)$ contains all sufficiently large integers. A semigroup having these properties is called a numerical semigroup. It is observed in [3, Remark 3.11] that every numerical semigroup $S$ is realizable as $S(M)$, where $(R, M)$ is a local domain of altitude one. 
Definition 1.3 The set $\mathbf{P}(I)$ is said to be projectively full if $S(I)=\mathbb{N}_{0}$, or equivalently, if every element of $\mathbf{P}(I)$ is the integral closure of a power of the largest element $K$ of $\mathbf{P}(I)$, i.e., every element of $\mathbf{P}(I)$ has the form $\left(K^{n}\right)_{a}$, for some positive integer $n$. If this holds, then each ideal $J$ in $R$ such that $J_{a}=K$ is said to be projectively full.

Our main goals may be listed as follows:

1. Describe ideals $K$ that are projectively full.

2. Describe ideals $I$ such that $\mathbf{P}(I)$ is projectively full.

3. Determine if improvements can be obtained by passing to an integral extension ring.

In connection with the third main goal, the following theorem is proved in [4].

Theorem 1.4 If $R$ contains the field of rational numbers, then there exists a finite free integral extension ring $A$ of $R$ such that $\mathbf{P}(I A)$ is projectively full; and if $R$ is an integral domain, then there also exists a finite integral extension domain $B$ of $R$ such that $\mathbf{P}(I B)$ is projectively full.

A strengthened version of Theorem 1.4 is given in Corollary 7.5 below.

Examples 1.5 and 1.6 illustrate aspects of the projectively full property.

Example 1.5 Let $(R, M)$ be a Noetherian local ring having the property

$$
a \in M^{i} \backslash M^{i+1} \text { and } b \in M^{j} \backslash M^{j+1} \Longrightarrow a b \notin M^{i+j+1}
$$

then $M$ is projectively full. Thus if the associated graded ring

$$
\mathbf{G}(R, M)=R / M \oplus M / M^{2} \oplus \cdots \oplus M^{n} / M^{n+1} \oplus \cdots
$$

is an integral domain, then $M$ is projectively full.

Example 1.6 Let $x$ be an indeterminate over the field $F$ and let $R=F\left[\left[x^{2}, x^{3}\right]\right]$. Then the maximal ideal $M=\left(x^{2}, x^{3}\right) R$ is not projectively full. The numerical semigroup $S(M)$ is generated by 2 and 3 . 
Notice that with $R=F\left[\left[x^{2}, x^{3}\right]\right]$ as in Example [1.6, the $\operatorname{ring} R$ is not integrally closed. Things improve by passing to the integral extension ring $R[x]=F[[x]]$. Indeed, for each regular proper ideal $I$ of $R, \mathbf{P}(I R[x])$ is projectively full.

Example 1.7 is established in 3] to demonstrate existence of a normal local domain $(R, M)$ such that $M$ is not projectively full.

Example 1.7 Let $F$ be a field in which 2 and 3 are units and let $x, y, z, w$ be variables. Let

$$
R_{0}=F[x, y]_{(x, y)} \quad \text { and } \quad R=\frac{R_{0}[z, w]}{\left(z^{2}-x^{3}-y^{3}, w^{2}-x^{3}+y^{3}\right)} .
$$

Then $R$ is a normal local domain of altitude 1 two with maximal ideal $M=(x, y, z, w) R$, and $M$ is not projectively full.

\section{Some history.}

The concept of projective equivalence of ideals and the study of ideals projectively equivalent to $I$ was introduced by Samuel in [17] and further developed by Nagata in [14]. Rather than 'projectively equivalent', Hartmut Göhner uses the term 'asymptotically equivalent' in [6]. Göhner mentions that the expression 'projective asymptotic equivalence' is used by David Rees in [16] and by H. T. Muhly in [13].

Let $I$ be a regular proper ideal of a Noetherian $\operatorname{ring} R$. For each $x \in R$, let $v_{I}(x)=$ $\max \left\{k \in \mathbb{N} \mid x \in I^{k}\right\}$, and define

$$
\bar{v}_{I}(x)=\lim _{k \rightarrow \infty}\left(\frac{v_{I}\left(x^{k}\right)}{k}\right) .
$$

Rees established that:

(a) $\bar{v}_{I}(x)$ is well defined.

(b) For each $k \in \mathbb{N}$ and $x \in R, \bar{v}_{I}(x) \geq k$ if and only if $x \in\left(I^{k}\right)_{a}$.

(c) There exist discrete valuations $v_{1}, \ldots, v_{n}$ defined on $R$, (with values in $\mathbb{N} \cup \infty$ ) and positive integers $e_{1}, \ldots, e_{n}$ such that, for each $x \in R, \bar{v}_{I}(x)=\min \left\{\frac{v_{i}(x)}{e_{i}} \mid i=1, \ldots, n\right\}$.

\footnotetext{
${ }^{1}$ We are following Nagata [15] and using altitude for what is often now termed dimension or Krull dimension.
} 
In (c), we say that $v$ is a valuation on $R$ if $\{x \in R \mid v(x)=\infty\}$ is a prime ideal $P$, $v(x)=v(y)$ if $x+P=y+P$, and the function $v$ induces on $R / P$ is a valuation.

For simplicity, assume $R$ is a Noetherian integral domain with field of fractions $F$. Let $t$ be an indeterminate. The Rees ring of $R$ with respect to $I$ is the graded subring

$$
\mathbf{R}=R\left[t^{-1}, I t\right]=\bigoplus_{n \in \mathbb{Z}} I^{n} t^{n}
$$

of the Laurent polynomial ring $R\left[t^{-1}, t\right]$. The integral closure $B$ of $R\left[t^{-1}, I t\right]$ is a Krull domain, and $B_{P}$ is a DVR for each minimal prime $P$ of $t^{-1} B$. The set Rees $I$ of Rees valuation rings of $I$ is precisely the set of rings $V=B_{P} \cap F$, where $P$ is a minimal prime of $t^{-1} B$.

Definition 2.1 Let $\left(V_{1}, N_{1}\right), \ldots,\left(V_{n}, N_{n}\right)$ be the Rees valuation rings of $I$. The integers $\left(e_{1}, \ldots, e_{n}\right)$, where $I V_{i}=N_{i}^{e_{i}}$, are the Rees integers of $I$. (These are the same $e_{i}$ as in (c) above.)

A sufficient condition for $I$ to be projectively full is that $\operatorname{gcd}\left(e_{1}, \ldots, e_{n}\right)=1$. However, this is not a necessary condition as we demonstrate in Example 3.1 .

\section{Projective equivalence and Rees valuation rings.}

Example 3.1 Let $(R, M)$ be a regular local ring of altitude two with $M=(x, y) R$, and let $e>1$ be an integer. The ideal $I=\left(x, y^{e}\right) R$ is integrally closed with unique Rees valuation ring $V=R\left[x / y^{e}\right]_{M R\left[x / y^{e}\right]}$. The integrally closed ideals projectively equivalent to $I$ are precisely the powers $I^{n}$ of $I$. Thus $I$ is projectively full. The maximal ideal of $V$ is $N=y V$ and $I V=N^{e}$, so the gcd of the Rees integers of $I$ is $e>1$.

If $I$ and $J$ are projectively equivalent, then Rees $I=$ Rees $J$. The converse holds if $I$ or $J$ has only one Rees valuation ring, but fails in general. Steve McAdam, Jack Ratliff and Judy Sally show in [11 that if $I$ and $J$ are projectively equivalent, then the Rees integers of $I$ and $J$ are proportional. It is observed in [2] that the converse also holds: if Rees $I=$ Rees $J$ and the Rees integers of $I$ and $J$ are proportional, then $I$ and $J$ are projectively equivalent. 
Example 3.2 Assume $(R, M)$ is a regular local ring of altitude two. Zariski's theory of unique factorization of complete (= integrally closed) ideals of $R$ as finite products of simple complete ideals implies $\mathbf{P}(I)$ is projectively full for each nonzero proper ideal $I$ of $R$. The ideal $I$ has a unique Rees valuation ring if and only if $I$ is a power of a simple complete ideal. If $I$ factors as $I=I_{1}^{f_{1}} \cdots I_{n}^{f_{n}}$, where $I_{1}, \ldots, I_{n}$ are distinct simple complete ideals, then $I$ is projectively full if and only if $\operatorname{gcd}\left(f_{1}, \ldots, f_{n}\right)=1$.

How do the integers $f_{1}, \ldots, f_{n}$ relate to the Rees integers of $I$ ? The simple complete $M$-primary ideals of $R$ are in one-to-one correspondence with the prime divisors birationally dominating $R$. Thus the Rees valuation rings of $I$ are $\left(V_{1}, N_{1}\right), \ldots,\left(V_{n}, N_{n}\right)$, where $\left(V_{j}, N_{j}\right)$ corresponds to $I_{j}$. If $I_{j} V_{j}=N_{j}^{c_{j}}$, then the Rees integers of $I$ are $e_{1}=c_{1} f_{1}, \ldots, e_{n}=c_{n} f_{n}$.

Example 3.3 illustrates these concepts in a specific situation.

Example 3.3 Assume $(R, M)$ is a regular local ring of altitude two with $M=(x, y) R$ as in Example 3.1. Let $I=\left(x^{2}, x y^{2}, y^{3}\right) R$. Notice that $J=\left(x^{2}, y^{3}\right) R$ is a reduction of $I$ and $J I=I^{2}$, so the reduction number $r_{J}(I)=1$. Let

$$
V=R\left[\frac{x y^{2}}{x^{2}}, \frac{y^{3}}{x^{2}}\right]_{M R\left[\frac{x y^{2}}{x^{2}}, \frac{y^{3}}{x^{2}}\right]}=R\left[\frac{y^{2}}{x}, \frac{y^{3}}{x^{2}}\right]_{M R\left[\frac{y^{2}}{x}, \frac{y^{3}}{x^{2}}\right.} .
$$

One sees that $V$ is a valuation ring with maximal ideal $N=\left(y^{2} / x\right) V$, and $I$ is a simple complete ideal. The ideals of $R$ that are contracted from $V$ descend as follows: $M=$ $N^{2} \cap R \supsetneq\left(x, y^{2}\right) R=N^{3} \cap R \supsetneq M^{2}=N^{4} \cap R \supsetneq\left(x, y^{2}\right) M=N^{5} \cap R \supsetneq I=N^{6} \cap R$. The ideals in $\mathbf{P}(I)$ are precisely the ideals $I^{m}=N^{6 m} \cap R$, for $m \in \mathbb{N}$.

\section{The Rees integers and the associated graded ring.}

The following result is established in [4, Example 5.1].

Proposition 4.1 If $\mathbf{G}(R, I)=R / I \oplus I / I^{2} \oplus \cdots \oplus I^{n} / I^{n+1} \oplus \cdots$ has a minimal prime $z$ such that $z$ is its own z-primary component of (0), then I has a Rees integer equal to one. Therefore $I$ is projectively full. In particular, if $\mathbf{G}(R, I)$ is reduced, then $I$ is projectively full. 
More can be said about the Rees integers of $I$ by using the Rees ring $\mathbf{R}=R\left[t^{-1}, I t\right]$ and the identification $\mathbf{G}(R, I)=\mathbf{R} / t^{-1} \mathbf{R}$. Let $\mathbf{R}^{\prime}$ denote the integral closure of $\mathbf{R}$. Proposition 4.2 is from [4, Proposition 4.1].

Proposition 4.2 The ideal I has a Rees integer equal to one if and only if $t^{-1} \mathbf{R}$ has a minimal prime $p$ such that $t^{-1} \mathbf{R}_{p}^{\prime}=p \mathbf{R}_{p}^{\prime}$.

Let $(R, M)$ be a normal local domain and let $\mathbf{G}(R, M)$ denote the associated graded ring of $R$ with respect to $M$. Proposition 4.1 implies that $M$ is projectively full if $\mathbf{G}(R, M)$ is reduced. Example 4.3 shows that the converse of this statement is not true.

Example 4.3 An example of a normal local domain $(R, M)$ of altitude two such that $M$ is projectively full and the associated graded $\operatorname{ring} \mathbf{G}(R, M)$ is not reduced. Let $F$ be an algebraically closed field with char $F=0$, and let $R_{0}$ be a regular local domain of altitude two with maximal ideal $M_{0}=(x, y) R_{0}$ and coefficient field $F$, e.g., $R_{0}=F[x, y]_{(x, y)}$, or $R_{0}=F[[x, y]]$. Then $V_{0}=R_{0}[y / x]_{x R_{0}[y / x]}$ is the unique Rees valuation ring of $M_{0}$. Let $R=R_{0}[z]$, where $z^{2}=x^{3}+y^{j}$ with $j \geq 3$. It is readily checked that $R$ is a normal local domain of altitude two with maximal ideal $M=(x, y, z) R$. Moreover, the image of $z$ in $M / M^{2}$ is a nonzero nilpotent element of the associated graded $\operatorname{ring} \mathbf{G}(R, M)$, so $\mathbf{G}(R, M)$ is not reduced.

Notice that $I=(x, y) R$ is a reduction of $M$ since $z$ is integral over $I$. It follows that every Rees valuation ring of $M$ is an extension of $V_{0}$. Let $V$ be a Rees valuation ring of $M$ and let $v$ denote the normalized valuation with value group $\mathbb{Z}$ corresponding to $V$. Then $v(x)=v(y)$ and the image of $y / x$ in the residue field of $V$ is transcendental over $F$. Since $z^{2}=x^{3}+y^{j}$ and $j \geq 3$, we have

$$
2 v(z)=v\left(z^{2}\right)=v\left(x^{3}+y^{j}\right)=3 v(x) .
$$

We conclude that $v(x)=2$ and $v(z)=3$. Therefore $V$ is ramified over $V_{0}$. This implies that $V$ is the unique extension of $V_{0}$ in the quadratic field extension generated by $z$ over the field of fractions of $R_{0}$, and thus the unique Rees valuation ring of $M$.

For each positive integer $n$, let $I_{n}=\{r \in R \mid v(r) \geq n\}$. Thus $I_{2}=M$. Since $V$ is the unique Rees valuation ring of $M$, we have $I_{2 n}=\left(M^{n}\right)_{a}$ for each $n \in \mathbb{N}$. 
To show $M$ is projectively full, we prove that $V$ is not the unique Rees valuation ring of $I_{2 n+1}$ for each $n \in \mathbb{N}$. Consider the inclusions

$$
M^{2} \subseteq I_{4} \subset\left(z, x^{2}, x y, y^{2}\right) R:=J \subseteq I_{3} \subset I_{2}=M
$$

Since $\lambda\left(M / M^{2}\right)=3$ and since the images of $x$ and $y$ in $M / M^{2}$ are $F$-linearly independent, $J=I_{3}$ and $M^{2}=I_{4}=\left(M^{2}\right)_{a}$.

Since $x^{3}=z^{2}-y^{j}$ and $j \geq 3, L=\left(z, y^{2}\right) R$ is a reduction of $I_{3}=\left(z, x^{2}, x y, y^{2}\right) R$. Indeed, $\left(x^{2}\right)^{3} \in L^{3}$ and $(x y)^{3} \in L^{3}$ implies $x^{2}$ and $x y$ are integral over $L$. It follows that $V$ is not a Rees valuation of $I_{3}$, for $z V \neq y^{2} V$.

Consider $M^{3} \subset I_{3} M \subseteq I_{5} \subset I_{4}=M^{2}$. Since the images of $x^{2}, x y, y^{2}, x z, y z$ in $M^{2} / M^{3}$ are an $F$-basis, it follows that $I_{3} M=I_{5}$ and $M^{3}=\left(M^{3}\right)_{a}=I_{6}$. Proceeding by induction, we assume $M^{n+1}=\left(M^{n+1}\right)_{a}=I_{2 n+2}$, and that $I_{2 n+1}$ is not projectively equivalent to $M$. Consider

$$
M^{n+2} \subset I_{3} M^{n} \subseteq I_{2 n+3} \subset I_{2 n+2}=M^{n+1} .
$$

Since the images in $M^{n+1} / M^{n+2}$ of $\left\{x^{a} y^{b} \mid a+b=n+1\right\} \cup\left\{z x^{a} y^{b} \mid a+b=n\right\}$ is an $F$-basis, $\lambda\left(M^{n+1} / M^{n+2}\right)=2 n+3$, and the inequalities $\lambda\left(M^{n+1} / I_{2 n+3}\right) \geq n+2$ and $\lambda\left(I_{3} M^{n} / M^{n+2}\right) \geq n+1$ imply $I_{3} M^{n}=I_{2 n+3}$ and $M^{2 n+2}=\left(M^{2 n+2}\right)_{a}$.

Therefore the ideal $I_{2 n+3}$ has a Rees valuation ring different from $V$, and thus is not projectively equivalent to $M$. We conclude that $M$ is projectively full. We have also shown that $M$ is a normal ideal.

\section{Questions and examples in altitude two.}

In Questions 5.1 we list several questions that interest us and that we hope may stimulate further research work in this area.

Questions 5.1 Let $(R, M)$ be a complete normal local domain of altitude two.

1. What are necessary and sufficient conditions in order that $M$ be projectively full?

2. Let $I$ be an $M$-primary ideal. What are necessary and sufficient conditions in order that $I$ be projectively full? 
3. What are necessary and sufficient conditions in order that $\mathbf{P}(I)$ be projectively full for each $M$-primary ideal $I$ of $R$ ?

4. What are necessary and sufficient conditions in order that $\mathbf{P}(I)$ be projectively full for each nonzero proper ideal $I$ of $R$ ?

In Example 5.2, we present in more detail a family of examples from [3, Example 5.1] that includes Example 1.7.

Example 5.2 An example of a (complete) normal local domain $(R, M)$ of altitude two such that $M$ is not projectively full. Let $F$ be an algebraically closed field, and let $R_{0}$ be a regular local domain of altitude two with maximal ideal $M_{0}=(x, y) R_{0}$ and coefficient field $F$, e.g., $R_{0}=F[x, y]_{(x, y)}$, or $R_{0}=F[[x, y]]$. Let $k<i$ be relatively prime positive integers $\geq 2$, and let $R=R_{0}[z, w]$, where $z^{k}=x^{i}+y^{i}$ and $w^{k}=x^{i}-y^{i}$. Assume that $2, i$ and $k$ are units of $F$. It is readily checked that $R$ is a normal local domain of altitude two with maximal ideal $M=(x, y, z, w) R$. Also $R$ is a free $R_{0}$-module of rank $k^{2}$.

With $R=R_{0}[z, w]$ as above, we show that $M=(x, y, z, w) R$ has a unique Rees valuation ring and that $M$ is not projectively full. Notice that $L=(x, y) R$ is a reduction of $M$, for $z^{k} \in\left(x^{i}, y^{i}\right) R \subseteq L^{k}$ and $w^{k} \in\left(x^{i}, y^{i}\right) R \subseteq L^{k}$ imply $z$ and $w$ are integral over $L$. Thus each Rees valuation ring $V$ of $M$ is an extension of the order valuation ring $V_{0}=R_{0}[y / x]_{x R_{0}[y / x]}$ of $R_{0}$. To show there exists a unique Rees valuation ring of $M$, we observe that if $V$ is a Rees valuation ring of $M$, then $V$ as an extension of $V_{0}$ ramifies of degree $k$ and undergoes a residue field extension of degree at least $k$.

To show $V$ ramifies of degree $k$ over $V_{0}$, observe that $k v(z)=v\left(z^{k}\right)=v\left(x^{i}+y^{i}\right)=$ $i v(x)=i v(y)$ implies $v(z)=i$ and $v(x)=v(y)=k$. Similarly, $v(w)=i$. Let $N$ denote the maximal ideal of $V$. We have $(z, w) V=N^{i}$ and $M V=(x, y) V=N^{k}$. The residue field of $V_{0}$ is $F(\bar{\tau})$, where $\bar{\tau}$ is the image of $\tau=y / x$ and is transcendental over $F$. Now $w / z$ is a unit of $V$ and

$$
\left(\frac{w}{z}\right)^{k}=\frac{x^{i}-y^{i}}{x^{i}+y^{i}}=\frac{1-\tau^{i}}{1+\tau^{i}} .
$$

It follows that the residue class of $w / z$ in $V / N$ is algebraic of degree $k$ over $F(\bar{\tau})$. This proves that $V$ is the unique Rees valuation ring of $M$. 
To show $M$ is not projectively full, notice that $\left(z^{k}, w^{k}\right) R=\left(x^{i}+y^{i}, x^{i}-y^{i}\right) R$, and since $\operatorname{char} F \neq 2, \quad\left(x^{i}+y^{i}, x^{i}-y^{i}\right) R=\left(x^{i}, y^{i}\right) R . \quad$ Since $\left(x^{i}, y^{i}\right) R$ is a reduction of $M^{i}$, we have $\left(\left(z^{k}, w^{k}\right) R\right)_{a}=\left(M^{i}\right)_{a} . \quad$ Also $\left(\left(z^{k}, w^{k}\right) R\right)_{a}=\left((z, w)^{k} R\right)_{a}$. Therefore $(z, w) R$ and $M$ are projectively equivalent, so $V$ is the unique Rees valuation ring of $(z, w) R$, and $((z, w) R)_{a}=N^{i} \cap R$. We have $M=N^{k} \cap R, M^{n} V=N^{n k}$ and $\left(M^{n}\right)_{a}=N^{n k} \cap R$, for each positive integer $n$. Since $i$ is not a multiple of $k, M$ is not projectively full.

Remark 5.3 Let $(R, M)$ be a normal local domain of altitude two. Muhly and Sakuma in [12] define the filtration $\left\{A_{i} \mid i \in \mathbb{N}_{0}\right\}$ associated to a prime $R$-divisor $v$ by $A_{0}$ $=R$ and $A_{i}=\left\{x \in R \mid v(x)>v\left(A_{i-1}\right\}\right.$ for $i \geq 1$. The prime $R$-divisor $v$ is said to be Noetherian if the associated filtration $\left\{A_{i} \mid i \geq 0\right\}$ possesses a Noetherian subfiltration, that is a subfiltration $\left\{A_{i_{j}} \mid j \geq 0\right\}$ such that the graded ring $\oplus_{j \geq 0} A_{i_{j}} / A_{i_{j+1}}$ is Noetherian. Muhly and Sakuma say that a normal local domain $R$ satisfies condition $\mathbf{N}$ if each prime divisor of the second kind relative to $R$ is Noetherian [12, First sentence of Section 4]. They show that if $R$ is analytically normal of altitude two, then each prime divisor of the second kind relative to $R$ is the unique valuation ring of some ideal $I$ of $R$ if and only if $R$ satisfies condition N [12, Proposition 2.2 and the paragraph following it]. (It is shown in [1] that some caution should be used in reading the given proof of their needed result [12, Proposition 2.1].) Muhly and Sakuma also show in [12] that if an analytically normal local domain $R$ of altitude two satisfies condition $(\mathrm{N})$, then there is a factorization theory for complete $M$-primary ideals that extends some aspects of Zariski's factorization theory for complete ideals in the case where $R$ is regular. Muhly carries this work further in [13 by showing that the hypothesis that $R$ is analytically normal can be weakened to $R$ is normal, and also that contrary to what was conjectured in [12], $R$ being analytically normal does not imply $R$ satisfies condition $(\mathrm{N})$.

Assume $(R, M)$ is a complete normal local domain of altitude two. Göhner in [6] proves that $R$ satisfies condition $(\mathrm{N})$ if $R$ is semifactorial, i.e., if $\operatorname{Pic} R$ is a torsion group, and conjectures that the converse also holds. Cutkosky proves this conjecture in [5]. This establishes an interesting connection between the divisorial ideals of $R$ and the complete $M$-primary ideals. 
Göhner [6, Corollary 4.5] observes that if $(R, M)$ is a complete normal local domain of altitude two and if $R / M$ is the algebraic closure of a finite field, then $R$ is semifactorial and therefore satisfies condition $(\mathrm{N})$. By taking $F$ in Example 5.2 to be the algebraic closure of a finite field in which $2, i$ and $k$ are units, we see that there exists a complete normal local domain $(R, M)$ that satisfies condition $(\mathrm{N})$ and has the property that its maximal ideal $M$ is not projectively full.

Remark 5.4 Let $(R, M)$ be a normal local domain of altitude two and let $I$ be an $M$ primary ideal. Recall that the multiplicity $e(I)=e(I, R)$ of $I$ is a positive integer [10, Section 14]. In connection with Questions [5.1, Joe Lipman pointed out to us that if $e(I)$ is squarefree, then $I$ is projectively full. For an ideal and its integral closure have the same multiplicity, and for each positive integer $n, e\left(I^{n}\right)=n^{2} e(I)$ [10, Formula 14.3]. If $m$ and $n$ are relatively prime positive integers and $J$ is an ideal such that $\left(I^{m}\right)_{a}=\left(J^{n}\right)_{a}$, then $m^{2} e(I)=n^{2} e(J)$, and the assumption that $e(I)$ is squarefree implies that $n=1$. Therefore the hypothesis that $e(I)$ is squarefree implies that $I$ is projectively full.

More generally, let $(R, M)$ be a local domain of altitude $d \geq 2$ and let $I$ be an $M$ primary ideal. For each positive integer $n$, we have $e\left(I^{n}\right)=n^{d} e(I)$. Assume that $m$ and $n$ are relatively prime positive integers and that $J$ is an ideal such that $\left(I^{m}\right)_{a}=\left(J^{n}\right)_{a}$. Then $m^{d} e(I)=n^{d} e(J)$. If $e(I)$ is free of $d$-th powers, then $n=1$. Therefore the hypothesis that $e(I)$ is free of $d$-th powers implies that $I$ is projectively full.

Notice that for $(R, M)$ as in Example 5.2, we have $e(M)=k^{2}$. In particular, for each integer $k \geq 2$, Example 5.2 establishes the existence of a normal local domain $(R, M)$ of altitude two such that $M$ is not projectively full and $e(M)=k^{2}$.

\section{Rational singularities and the closed fiber.}

Lipman in his paper [9] extends Zariski's theory of complete ideals in a regular local domain of altitude two to the case where $R$ is a normal local domain of altitude two that has a rational singularity. Lipman proves that $R$ has unique factorization of complete ideals if and only if the completion of $R$ is a UFD. For $R$ having this property, it follows that $\mathbf{P}(I)$ is projectively full for each nonzero proper ideal $I$, e.g. this is the case if $R=F[[x, y, z]]$, 
where $z^{2}+y^{3}+x^{5}=0$ and $F$ is a field of characteristic zero.

Let $(R, M)$ be a normal local domain of altitude two. Göhner proves that if $R$ has a rational singularity, then the set of complete asymptotically irreducible ideals associated to a prime $R$-divisor $v$ consists of the powers of an ideal $A_{v}$ which is uniquely determined by $v$. In our terminology, this says that if $I$ is a nonzero proper ideal of $R$ having only one Rees valuation ring, then $\mathbf{P}(I)$ is projectively full. Göhner's proof involves choosing a desingularization $f: X \rightarrow \operatorname{Spec} R$ such that $v$ is centered on a component $E_{1}$ of the closed fiber on $X$.

Let $E_{2}, \ldots, E_{n}$ be the other components of the closed fiber on $X$. Let $E_{X}$ denote the group of divisors having the form $\sum_{i=1}^{n} n_{i} E_{i}$, with $n_{i} \in \mathbb{Z}$, and consider

$$
\begin{gathered}
E_{X}^{+}=\left\{D \in E_{X} \mid D \neq 0 \text { and }\left(D \cdot E_{i}\right) \leq 0 \text { for all } 1 \leq i \leq n\right\}, \text { and } \\
E_{X}^{\#}=\left\{D \in E_{X} \mid D \neq 0 \text { and } O(-D) \text { is generated by its sections over } X\right\} .
\end{gathered}
$$

Lipman shows $E_{X}^{\#} \subseteq E_{X}^{+}$and equality holds if $R$ has a rational singularity. Also, if $D=\sum_{i} n_{i} E_{i} \in E_{X}^{+}$, then negative-definiteness of the intersection matrix $\left(E_{i} \cdot E_{j}\right)$ implies $n_{i} \geq 0$ for all $i$.

For if $D \in E_{X}^{+}$and $D=A-B$, where $A$ and $B$ are effective, then $(A-B \cdot B) \leq 0$ and $(A \cdot B) \geq 0$ imply $(B \cdot B) \geq 0, \quad$ so $B=0$.

Let $v=v_{1}, v_{2}, \ldots, v_{n}$ denote the discrete valuations corresponding to $E_{1}, \ldots, E_{n}$. Associated with $D=\sum_{i} n_{i} E_{i} \in E_{X}^{\#}$ one defines the complete $M$-primary ideal

$$
I_{D}=\left\{r \in R \mid v_{i}(r) \geq n_{i} \text { for } 1 \leq i \leq n\right\}
$$

This sets up a one-to-one correspondence between elements of $E_{X}^{\#}$ and complete $M$ primary ideals that generate invertible $O_{X}$-ideals.

Lipman suggested to us the following proof that $\mathbf{P}(I)$ is projectively full for each complete $M$-primary ideal $I$ if $R$ has a rational singularity. Fix a desingularization

$$
f: X \rightarrow \operatorname{Spec} R
$$

such that $I$ generates an invertible $O_{X}$-ideal and let $D=\sum_{i} n_{i} E_{i} \in E_{X}^{\#}$ be the divisor associated to $I$. Let $g=\operatorname{gcd}\left\{n_{i}\right\}$. Since $E^{+}=E^{\#}$, it follows that $(1 / g) D \in E^{\#}$. 
The ideals $J \in \mathbf{P}(I)$ correspond to divisors in $E^{\#}$ that are integral multiples of $(1 / g) D$. Thus if $K$ is the complete $M$-primary ideal associated to $(1 / g) D$, then each $J \in \mathbf{P}(I)$ is the integral closure of a power of $K$, so $\mathbf{P}(I)$ is projectively full.

\section{$7 \quad$ Integral extensions.}

Perhaps the main unresolved question related to projectively full ideals is the following:

Question 7.1 Let $I$ be a nonzero ideal of a Noetherian domain $R$. Does there always exist a finite integral extension domain $B$ of $R$ such that $\mathbf{P}(I B)$ is projectively full?

An affirmative answer to Question [7.1] is obtained in [4, Theorem 2.5] if the ideal $I$ has a Rees integer that is a unit of $R$. More precisely, let $I=\left(b_{1}, \ldots, b_{g}\right) R$ be a nonzero regular ideal of the Noetherian ring $R$, let $R_{g}=R\left[X_{1}, \ldots, X_{g}\right]$ and $K=\left(X_{1}^{c}-b_{1}, \ldots, X_{g}^{c}-b_{g}\right) R_{g}$, where $c$ is a positive integer. Then $A=R_{g} / K$ is a finite free integral extension ring of rank $c^{g}$ of $R$. Let $x_{i}=X_{i} \bmod K$. Then $J=\left(x_{1}, \ldots, x_{g}\right) A$ is such that $(I A)_{a}=\left(J^{c}\right)_{a}$, so $I A$ and $J$ are projectively equivalent.

Theorem 7.2 Let $I=\left(b_{1}, \ldots, b_{g}\right) R$ be as above and let $(V, N)$ be a Rees valuation ring of $I$. Assume that $b_{i} V=I V=N^{c}$ for each $i$ with $1 \leq i \leq g$, and that $c$ is a unit of $R$. Then the finite free integral extension ring $A=R_{g} / K$ is such that $J=\left(x_{1}, \ldots, x_{g}\right) A$ is projectively full. Hence $\mathbf{P}(I A)$ is projectively full. If $R$ is an integral domain and $z$ is a minimal prime of $A$, then $B=A / z$ is an integral extension domain of $R$ such that $\mathbf{P}(I B)$ is projectively full.

Let $I$ be a regular proper ideal in a Noetherian $\operatorname{ring} R$ and let $e$ be the least common multiple of the Rees integers of $I$. Consider the Rees ring $R\left[t^{-1}, I t\right]$ and let $B$ denote the integral closure of $R\left[t^{-1}, I t\right]\left[t^{-\frac{1}{e}}\right]$ in $R\left[t^{-\frac{1}{e}}, t^{\frac{1}{e}}\right]$. Shiroh Itoh in [8, page 392] proves that $t^{-\frac{1}{e}} B$ is a radical ideal, and $B / t^{-\frac{1}{e}} B$ is a reduced graded ring.

This result of Itoh motivated us to ask about the existence of an integral extension ring $A$ of $R$ in which there exists a radical ideal that is projectively equivalent to $I A$. The following two result are established in [7]. 
Theorem 7.3 Let $I$ be a regular proper ideal in a Noetherian ring $R$ and let $b_{1}, \ldots, b_{g}$ be regular elements in $R$ that generate $I$. Let $m$ be a positive integer and let

$$
K=\left(X_{1}^{m}-b_{1}, \ldots, X_{g}{ }^{m}-b_{g}\right) R\left[X_{1}, \ldots, X_{g}\right]
$$

For $i=1, \ldots, g$ let $x_{i}$ be the residue class of $X_{i}$ modulo $K$, let $A=R\left[x_{1}, \ldots, x_{g}\right]$, and let $J=\left(x_{1}, \ldots, x_{g}\right)$ A. Then:

(17.3.1) $A$ is a finite free integral extension ring of $R$ and $\left(J^{m}\right)_{a}=(I A)_{a}$, so $J$ is projectively equivalent to $I A$.

(7.3,2) $\left(J^{k}\right)_{a} \cap R=I_{\frac{k}{m}}\left(=\left\{x \in R \mid \bar{v}_{I}(x) \geq \frac{k}{m}\right\}\right)$ for all positive integers $k$.

(17.3.3) If $e$ is the least common multiple of the Rees integers of $I$ and $m=e$, then $J_{a}$ is a radical ideal.

(7.3,4) If $R$ is an integral domain and if $z$ is a minimal prime ideal in $A$, then $(J+z) / z$ is projectively equivalent to $(I A+z) / z$, and if $m=e$ as in 7.3.3), then $\left(J_{a}+z\right) / z$ is a radical ideal.

Theorem 7.4 Let $I$ be a regular proper ideal in a Noetherian ring $R$, let $V_{1}, \ldots, V_{n}$ be the Rees valuation rings of $I$, and let $e_{1}, \ldots, e_{n}$ be the corresponding Rees integers of I. Assume that the least common multiple e of $e_{1}, \ldots, e_{n}$ is a unit in $R$ and that there exists a regular element $b$ in $I$ such that $b V_{i}=I V_{i}$ for $i=1, \ldots, n$.

(17.4.1) Let $b_{1}, \ldots, b_{g}$ be regular elements in $R$ that generate $I$, let $A=R\left[x_{1}, \ldots, x_{g}\right]$ (= $\left.R\left[X_{1}, \ldots, X_{g}\right] /\left(X_{1}^{e}-b_{1}, \ldots, X_{g}{ }^{e}-b_{g}\right)\right)$, and let $J=\left(x_{1}, \ldots, x_{g}\right) A$. Then $A$ is a finite free integral extension ring of $R, J_{a}=\operatorname{Rad}(I A)$ is a projectively full radical ideal that is projectively equivalent to $I A,(I A)_{a}=\left(J^{e}\right)_{a}$, and all Rees integers of $J$ are equal to one. (17.4,2) If $R$ is a Noetherian domain, then let $b_{1}=b, b_{2}, \ldots, b_{g}$ be a basis of $I$, for $i=$ $1, \ldots, g$ fix an e-th root $b_{i}{ }^{1 / e}$ of $b_{i}$ in an algebraic closure of the quotient field of $R$, and let $A=R\left[b_{1}{ }^{1 / e}, \ldots, b_{g}{ }^{1 / e}\right]$ and $J=\left(b_{1}{ }^{1 / e}, \ldots, b_{g}{ }^{1 / e}\right) A$. Then $A$ is a Noetherian domain that is a finite integral extension of $R, \operatorname{Rad}(I A)=J_{a}$ is a projectively full radical ideal that is projectively equivalent to $I A,(I A)_{a}=\left(J^{e}\right)_{a}$, and all Rees integers of $J$ are equal to one.

We have the following corollary to Theorem 7.4 
Corollary 7.5 Let $I$ be a regular nonzero ideal in a Noetherian ring $R$ and assume that $R$ contains the field of rational numbers. Then there exists a finite free integral extension ring $A$ of $R$ that contains a projectively full radical ideal $J$ that is projectively equivalent to IA and whose Rees integers are all equal to one. If $R$ is an integral domain, then for each minimal prime ideal $z$ in $A$ the ideal $(J+z) / z$ in $A / z$ is a projectively full radical ideal that is projectively equivalent to $(I A+z) / z$, and all Rees integers of $(J+z) / z$ are equal to one.

The following example indicates difficulties in establishing a result similar to Theorem 7.4 without the hypothesis that $e$ is a unit of $R$.

Example 7.6 Let $D=k[y]$, where $k$ is a field of characteristic two and $y$ is an indeterminate, and let $b=y^{2}(y-1)$. Then $D$ is a PID, and $I=b D$ has the two Rees integers $e_{1}=$ 1 (for its Rees valuation ring $D_{p_{1}}$, where $p_{1}=(y-1) D$ ) and $e_{2}=2$ (for its Rees valuation ring $D_{p_{2}}$, where $p_{2}=y D$ ), so the least common multiple of $e_{1}, e_{2}$ is $e=2$. Let $E$ be the integral closure of $D[x]$ in its quotient field, where $x=\sqrt{b}$. Then $E$ is a Dedekind domain and the Rees integer of $x E$ with respect to $E_{y E}$ is 2 , so $x E$ is not a radical ideal of $E$.

\section{References}

[1] W. Bishop, J. W. Petro, L. J. Ratliff, Jr., and D. E. Rush, Note on Noetherian filtrations, Communications in Algebra 17(2) (1989), 471-485.

[2] C. Ciuperca, W. J. Heinzer, L. J. Ratliff, Jr., and D. E. Rush, Projectively equivalent ideals and Rees valuations, J. Algebra 282 (2004), 140-156.

[3] C. Ciuperca, W. J. Heinzer, L. J. Ratliff, Jr., and D. E. Rush, Projectively full ideals in Noetherian rings, J. Algebra (to appear).

[4] C. Ciuperca, W. J. Heinzer, L. J. Ratliff, Jr., and D. E. Rush, Projectively full ideals in Noetherian rings (II), J. Algebra (to appear).

[5] S. D. Cutkosky, On unique and almost unique factorization of complete ideals II, Invent. Math. 98 (1989), 59-74. 
[6] H. Göhner, Semifactoriality and Muhly's condition $(N)$ in two-dimensional local rings, J. Algebra 34 (1975), 403-429.

[7] W. J. Heinzer, L. J. Ratliff, Jr., and D. E. Rush, Projectively full radical ideals in integral extension rings, J. Algebra 317 (2007), 333-350.

[8] S. Itoh, Integral closures of ideals generated by regular sequences, J. Algebra 117 (1988), 390-401.

[9] J. Lipman, Rational singularities, with applications to algebraic surfaces and unique factorization, Publ. Math. Inst. Hautes Études Sci. No 36 (1969), 195-279.

[10] H. Matsumura, Commutative Ring Theory, Cambridge studies in advanced Mathematics, No. 8, Cambridge University Press, Cambridge, 1986.

[11] S. McAdam, L.J. Ratliff, Jr. and J. Sally, Integrally closed projectively equivalent ideals, in Commutative Algebra, MSRI Pub. 15, 1988, 391-405

[12] H. T. Muhly and M Sakuma, Asymptotic factorization of ideals, J. London Math. Soc. 33 (1963), 341-350.

[13] H. T. Muhly, On the existence of asymptotically irreducible ideals, J. London Math. Soc. 40 (1965), 99-107.

[14] M. Nagata, Note on a paper of Samuel concerning asymptotic properties of ideals, Mem. Coll. Sci. Univ. Kyoto, Ser. A Math. 30 (1957), 165-175.

[15] M. Nagata, Local Rings, Interscience, John Wiley, New York, 1962.

[16] D. Rees, Valuations associated with ideals (II), J. London Math. Soc. 36 (1956), 221228.

[17] P. Samuel, Some asymptotic properties of powers of ideals, Annals of Math 56 (1952), $11-21$.

Department of Mathematics, North Dakota State University, Fargo, North Dakota 58105-5075 E-mail address: catalin.ciuperca@ndsu.edu 
Department of Mathematics, Purdue University, West Lafayette, Indiana 47909-1395

E-mail address: heinzer@math.purdue.edu

Department of Mathematics, University of California, Riverside, California 92521-0135

E-mail address: ratliff@math.ucr.edu

Department of Mathematics, University of California, Riverside, California 92521-0135

E-mail address: rush@math.ucr.edu 